\title{
Polarized fluorescent emission in uniaxial liquid crystals. The effect of intramolecular energy transfer and rotational Brownian motion on measurements of the orientational distribution function
}

\section{Chapoy, Larry Lawrence; DuPré, Donald B.}

Published in:

Journal of Chemical Physics

Link to article, DOI:

$10.1063 / 1.436642$

Publication date:

1978

Document Version

Publisher's PDF, also known as Version of record

Link back to DTU Orbit

Citation (APA):

Chapoy, L. L., \& DuPré, D. B. (1978). Polarized fluorescent emission in uniaxial liquid crystals. The effect of intramolecular energy transfer and rotational Brownian motion on measurements of the orientational distribution function. Journal of Chemical Physics, 69(2), 519-524. https://doi.org/10.1063/1.436642

\section{General rights}

Copyright and moral rights for the publications made accessible in the public portal are retained by the authors and/or other copyright owners and it is a condition of accessing publications that users recognise and abide by the legal requirements associated with these rights.

- Users may download and print one copy of any publication from the public portal for the purpose of private study or research.

- You may not further distribute the material or use it for any profit-making activity or commercial gain

- You may freely distribute the URL identifying the publication in the public portal 


\title{
Polarized fluorescent emission in uniaxial liquid crystals. The effect of intramolecular energy transfer and rotational Brownian motion on measurements of the orientational distribution function
}

\author{
L. Lawrence Chapoy and Donald B. DuPréa) \\ Instituttet for Kemiindustri, The Technical University of Denmark, DK-2800 Lyngby, Denmark \\ (Received 3 October 1977)
}

An expression is derived for the anisotropic fluorescent emission in uniaxial liquid crystals where fluorescent sites governed by an initial nonrandom distribution of orientations are subject to rotational Brownian motion. The possibility of nonparallelism of absorption and emission oscillators is also considered.

\section{INTRODUCTION}

The approach to the mathematical formulation of the distribution function that describes long range orientational ordering of molecules in liquid crystals is necessarily an approximation. Some workers propose model forms with one or more adjustable parameters used to fit data of some experimental property sensitive to an average over this unattainable function. These attempts are open to criticism of the reasonableness and uniqueness of the chosen form of the approximation. An alternative procedure, which is mathematically rigorous and unambiguous, is to write the function as a truncated series expansion whose coefficients are experimentally determinable moments of the real distribution. This method is usually limited by the availability of only the second moment. Raman, ${ }^{1-5}$ magnetic resonance, ${ }^{6}$ and fluorescent emission spectroscopy ${ }^{1+2}$ are capable of supplying both the second and fourth moments, hence extending the expansion and making it a more faithful representation of the real distribution. The fluorescence technique, however, has inherent complications in that there is usually a significant delay ( $1-10 \mathrm{nsec}$ ) between the absorption and emission process and emitted radiation may emanate polarized along a different axis of the excited molecule. We consider below the effect of rotational Brownian motion of the fluorescent site during the lifetime of the excitation and the nonparallelism of absorption and emission oscillators. An expression is derived for the anisotropy of emission which contains the possibility of a nonrandom distribution of fluorescent sites before the photoselection ${ }^{7,8}$ process. A nonzero intramolecular energy transfer angle $\delta$ is included; and rotational relaxation appears through a reduced variable $\tau / \tau_{R}$, the ratio of the fluorescence lifetime to the rotational relaxation. time. Limits are considered and it is shown that considerable misinterpretation of experimental data, and hence the form of the orientational distribution function, can occur if the analysis of the experiment is not properly placed in the regime of $\delta$ and $\tau / \tau_{R}$.

\footnotetext{
a) On leave from the Department of Chemsitry, University of Louisville, Louisville, KY 40208.
}

\section{MOLECULAR ORDER IN THE LIQUID CRYSTAL: THE LONG RANGE ORIENTATIONAL DISTRIBUTION FUNCTION}

Consider a macroscopically aligned liquid crystal situated such that the major symmetry axes of the specimen are parallel to a laboratory based coordinate frame 0$X Y Z$. A representative molecule whose unique symmetry axis (long axis of the liquid crystal molecule) is denoted by $\mathbf{M}$ can be located in this coordinate system by polar and azimuthal angles $\theta, \phi$ as in Fig. 1. In discussing the orientational order of a collection of such molecules it is convenient to introduce a distribution function $f(\theta, \phi)$, relating the vectors $M$ to the macroscopic coordinates of the specimen. In many liquid crystals only uniaxial symmetry about the director is present and we only need consider an abbreviated orientation distribution $f(\theta)$.

In liquid crystal research it is the function $f(\theta)$ that is frequently sought as it completely describes the long range and anisotropic orientational order that gives these fluids their unique physical properties. (Uniaxial case

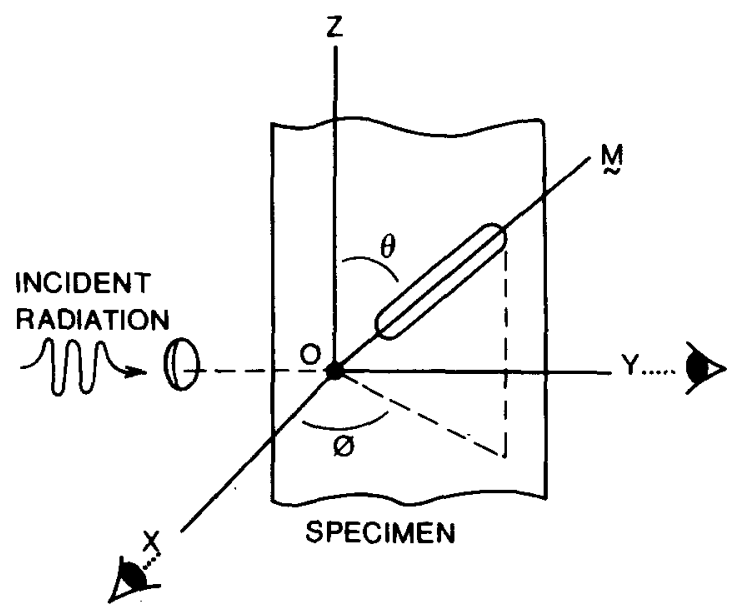

FIG. 1. Orientation of a representative molecule with unique axes $M$ in the laboratory frame $0-X Y Z$. The specimen is assumed to lie with its major symmetry axis along $Z$. Incident radiation enters from the left and travels along the $Y$ axis. The most common conditions of observation straight through $\left(\gamma=180^{\circ}\right)$ and at right angle $\left(\gamma=90^{\circ}\right)$ are indicated. 
assuming also that the liquid crystal molecule is a rigid entity and that molecular orientational order is thus specifiable in terms of the distribution of a single angle $\theta$. ) The significance of $f(\theta)$ is to liquid crystals as the single particle distribution function is to "normal" liquids. It is equally as difficult to obtain. The function, however, may be reproduced with accuracy sufficient for most purposes by a truncated series expansion ${ }^{9-11}$ involving the Legendre polynomials $P_{l}(\cos \theta)$ :

$$
\begin{aligned}
f(\theta) & =\sum^{\infty} \frac{2 l+1}{2}\left\langle P_{l}(\cos \theta)\right\rangle \cdot P_{l}(\cos \theta), \\
l & =0 \\
& \text { even }
\end{aligned}
$$

where the brackets indicate the statistical average of the quantity over all solid angles weighted by the distribution function $f(\theta)$. (A bar will be used interchangeably with $\langle\circ\rangle\rangle$ below. $)$ The first few terms of the expansion include the moments $\overline{\cos ^{2} \theta}$ and $\overline{\cos ^{4} \theta}$ as

$$
\begin{aligned}
& \left\langle P_{0}\right\rangle=1, \\
& \left\langle P_{2}\right\rangle=\frac{1}{2}\left(3 \overline{\cos ^{2} \theta}-1\right), \\
& \left\langle P_{4}\right\rangle=\frac{1}{8}\left(35 \overline{\cos ^{4} \theta}-30 \overline{\cos ^{2} \theta}+3\right) .
\end{aligned}
$$

The average of the second Legendre polynomial $\left\langle P_{2}\right\rangle$ is usually reported as $S$, the liquid crystal order parameter, ${ }^{12}$ but it is seen that $\left\langle P_{4}\right\rangle$ contains further information about orientational order present and should in fact be more sensitive to molecular fluctuations because of the higher powers of the deviation angle.

Although $\mathrm{x}$-ray scattering can in principle measure all the moments of the distribution $f(\theta)$, in practice it is limited by the lack of definite repetition of crystalline or der in liquid crystals. Optical birefringence; ir, visible, and uv dichroic measurements; and magnetic resonance techniques are the most frequently cited means of determining the degree of liquid crystalline order. The optical methods are inherently limited to only a measure of $\overline{\cos ^{2} \theta}$, while both the second and fourth moments are available from suitable analysis of magnetic resonance data $^{6}$ and polarized Raman ${ }^{1-5}$ and fluorescent emission ${ }^{1.2}$ spectra of active sites (embedded probes or sensitive molecular elements) in the liquid crystal. Hence, a description of $f(\theta)$ up to the third term of the series Eq. (1) is possible.

\section{THE POLARIZED FLUORESCENCE EXPERIMENT}

Fluorescent emission has many things in common with the Raman scattering as it is a two step spectroscopic process modulated by molecular motion. The singular difference is that, on the scale of time, the Raman effect is instantaneous (at least as fast as a molecular vibration of $\sim 10^{-12} \mathrm{sec}$ ) whereas fluorescent emission may follow absorptive excitation by many nanoseconds, perhaps even emanating from a different direction in the molecule. These factors lead to experimental and interpretative difficulties particularly when relaxation processes occur. In the following we will treat separately the complications of:

(i) nonparallelism of the absorption and emission oscillators at the fluorescent site, (ii) rotational Brownian motion of the excited molecules during the lifetime of the excitation $\tau$.

A method of analysis will be suggested for the determination of $\overline{\cos ^{2} \theta}$ and $\overline{\cos ^{4} \theta}$ by fluorescence spectrometry in liquid crystals and hence $f(\theta)$ to the second nontrivial term of Eq. (1).

Most fluorescent emission experiments are performed in the right angle geometry with the polarization of the incident and exit beam parallel or perpendicular to some symmetry axis of the specimen. We will restrict our discussion to this case here, letting the incident radiation propagate along the $Y$ axis from the left in vertical ( $Z$ axis) polarization. Observations are made along the $X$ axis with the analyzer in either the vertical (parallel) or horizontal (perpendicular) position (see Fig. 1). The observed fluorescence intensity depends on the square of the projection of the electric vector of the incident light on that of the absorption oscillator of the molecule and the square of the component of the emission oscillator radiation that projects on to and eventually emerges from the analyzer. In the fluorescence experiment, analysis of radiative capture and re-emission may be complicated by the factors listed above so the geometry and mobility of the active molecule must be considered in more detail. Figure 2 illustrates the general situation at the site of a cylindrically symmetric liquid crystal molecule or fluorescent probe molecule of similar geometry embedded in the medium. (It is assumed in the latter case that the presence of small quantities of fluorescent probes does not perturb the structure to be monitored and that the induced alignment of such guest molecules adequately reflects the order of the host.)

Letting $a$ and $e$ be unit vectors along the absorption and emission moments, respectively, Fig. 2 defines polar angles $\alpha$ and $\epsilon$ of their positions with respect to the long axis of the molecule $\mathbf{M}$. The azimuthal angles $\beta$ and $\beta+v$ are random, though connected, variables under the supposed cylindrical symmetry of the molecule. They are, however, significant degrees of freedom except when $\alpha$ or $\epsilon$ equals zero. $\delta$ is the intramolecular angle of energy transfer between the absorption and emission dipoles,

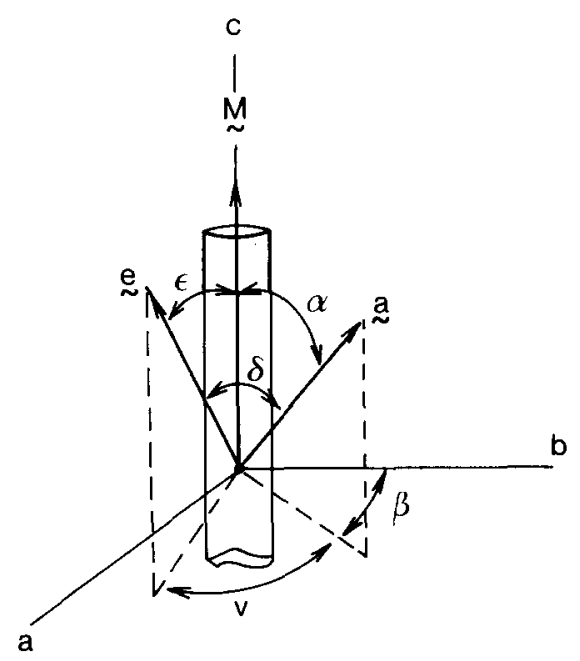

FIG. 2. Orientation of absorption and emission moments a and $e$, respectively, in the molecular frame $0-a b c$. 
where $\cos \delta=\mathbf{a} \cdot \mathbf{e}$, and may be regarded as a fixed, structural property of the molecule. The directions of the transition moments a and e relative to $M$ are in principle dependent on the wavelength of excitation and emission viewed. There are a number of fluorescent molecules which have a rodlike character to their overall geometry where the absorption moment is nearly parallel to the long molecular axis. It has been shown ${ }^{13,14}$ for example that in linear conjugated large molecules the low energy, strongly allowed absorption is due to a singlet electronic transition with transition moment in the direction of the long axis of the conjugated chain. We will assume this to be the case here and set $\alpha=0^{\circ}$ in the following. Even in this simplifying situation, however, the intramolecular angle $\delta$ will in general not be zero, and will thus be retained as it can considerably modify the interpretation of data.

The intensity of light emitted that is observed emerging through a polarization analyzer placed directly along a laboratory polarization axis $j$, resulting from light absorbed from an excitation source polarized directly along laboratory axis $i$, is given by ${ }^{9,15}$

$$
I_{i j}=\frac{1}{(2 \pi)^{2}} \int_{0}^{2 \pi} \int_{0}^{2 \pi} \int_{0}^{\pi} M_{a i}^{2} M_{e j}^{2} f(\theta) \sin \theta d \theta d \phi d \beta,
$$

where $M_{a i}^{2}$ and $M_{e f}^{2}$ are the squares of the absorption and emission oscillator components projected onto the laboratory axis $i$ and $j$, respectively. Instrumental, concentration, and volume factors have been incorporated into the definition of each $I_{i j}$, which are thus reduced intensities. It is the effect of the geometric disposition of fluorescent molecules that we seek to clarify. In general,

$$
\begin{aligned}
& M_{a t}^{2}=\left[\mathbf{a} \cdot \mathbf{0} \cdot \mathbf{u}_{\mathfrak{t}}\right]^{2}, \\
& M_{e j}^{2}=\left[\mathbf{e} \cdot \mathbf{0} \cdot \mathbf{u}_{\mathfrak{j}}\right]^{2},
\end{aligned}
$$

where $u_{i}$ is a unit vector pointing in the $i$ th direction in the laboratory frame and $a$ and $e$ are given, respectively, by

$$
\begin{aligned}
& \mathbf{a}=(\sin \alpha \sin \beta, \sin \alpha \cos \beta, \cos \alpha), \\
& \mathbf{e}=[\sin \epsilon \sin (\beta+v), \sin \epsilon \cos (\beta+v), \cos \epsilon]
\end{aligned}
$$

in the molecular frame. The rotational transformation matrix

$$
0=\left(\begin{array}{lll}
\cos \theta \cos \phi & \cos \theta \sin \phi & -\sin \theta \\
-\sin \phi & \cos \phi & 0 \\
\sin \theta \cos \phi & \sin \theta \sin \phi & \cos \theta
\end{array}\right)
$$

serves to bring the two tilted coordinate frames into coincidence.

For the fluorescence experiment performed in the right angle geometry with vertically polarized radiation we require products of

$$
M_{a z}^{2}=\cos ^{2} \theta
$$

and

$$
\begin{aligned}
M_{e y}^{2}= & \frac{1}{2} \cos ^{2} \theta \sin ^{2} \phi \sin ^{2} \delta \\
& +\sin ^{2} \theta \sin ^{2} \phi \cos ^{2} \delta+\frac{1}{2} \cos ^{2} \phi \sin ^{2} \delta,
\end{aligned}
$$

$$
M_{e z}^{2}=\frac{1}{2} \sin ^{2} \theta \sin ^{2} \delta+\cos ^{2} \theta \cos ^{2} \delta
$$

in the integrand of Eq. (3). [In Eqs. (9) and (10) the random variable $\beta$ has already been averaged out. ]

For a static collection of nonrandom molecules, Eqs. (3) and (8) $-(10)$ yield

$$
\begin{aligned}
I_{I} & =I_{z z}=\cos ^{2} \delta \cdot \overline{\cos ^{4} \theta}+\frac{1}{2} \sin ^{2} \delta \cdot\left(\overline{\cos ^{2} \theta}-\overline{\cos ^{4} \theta}\right), \\
I_{\perp} & =I_{z y}=\frac{1}{2} \cos ^{2} \delta \cdot\left(\overline{\cos ^{2} \theta}-\overline{\cos ^{4} \theta}\right) \\
& +\frac{1}{4} \sin ^{2} \delta \cdot\left(\overline{\cos ^{2} \theta}+\overline{\cos ^{4} \theta}\right),
\end{aligned}
$$

where $I_{11}$ and $I_{\perp}$ are the intensities measured with polars parallel and perpendicular, respectively. It is seen that information about $\overline{\cos ^{4} \theta}$ as well as $\overline{\cos ^{2} \theta}$ is available from measurements of these intensities.

\section{DEGREE OF POLARIZATION AND EMISSION ANISOTROPY}

The extent of the partially polarized fluorescent emission is frequently quoted in terms of one of two parameters measured in the right angle geometry: $p$, the degree of polarization, or $r$, the emission anisotropy defined through ${ }^{16,17}$

$$
p=\frac{I_{11}-I_{1}}{I_{11}+I_{\perp}}=\frac{I_{z z}-I_{z y}}{I_{z z}+I_{z y}}
$$

and

$$
r=\frac{I_{11}-I_{\perp}}{I_{11}+2 I_{\perp}}=\frac{I_{z \varepsilon}-I_{z y}}{I_{z z}+2 I_{z y}} .
$$

For a randomly oriented collection of immobile molecules whose absorption and emission moments are parallel, Eqs. (11) and (12) give $p_{0}=1 / 2, r_{0}=2 / 5$, where the subscript denotes this special case. These values obtain irrespective of the angle a or e makes with $\mathbf{M}$ so long as they remain colinear and immobile during the lifetime of the fluorescence process. If a and e form a fixed, nonzero intramolecular angle $\delta$, the polarization of emission is reduced and it can be shown also from Eqs. (11) and (12) that

$$
p_{0}=\frac{3 \cos ^{2} \delta-1}{3+\cos ^{2} \delta}, \quad r_{0}=\frac{3 \cos ^{2} \delta-1}{5}
$$

Equations (15) are therefore intrinsic polarization and anisotropy factors internal to the molecule, which may be measured in the isotropic phase of the liquid crystal quenched to freeze out thermal motions that also act to reduce the polarization (see next section).

The range of possible values which $p_{0}$ and $r_{0}$ can assume are

$$
-\frac{1}{3} \leqslant p_{0} \leqslant \frac{1}{2} ; \quad-\frac{1}{5} \leqslant r_{0} \leqslant \frac{2}{5},
$$

as $\delta$ may vary between 0 and $\pi / 2$.

Equations (15) may be alternatively written in a form where a multiplicative factor involving the second Legendre polynomial of $\cos \delta$ appears:

$$
\begin{aligned}
& 1 / p_{0}-\frac{1}{3}=\frac{5}{3}\left(\frac{2}{3 \cos ^{2} \delta-1}\right)=\frac{5}{3}\left[P_{2}(\cos \delta)\right]^{-1}, \\
& r_{0}=\frac{2}{5}\left(\frac{3 \cos ^{2} \delta-1}{2}\right)=\frac{2}{5} P_{2}(\cos \delta)
\end{aligned}
$$


If the distribution of molecular orientations is not random but otherwise static, Eqs. (11) and (12) yield

$$
r=\left(\frac{9 \overline{\cos ^{4} \theta}-3 \overline{\cos ^{2} \theta}}{6 \overline{\cos ^{2} \theta}}\right) \cdot P_{2}(\cos \delta) \text {. }
$$

The term in parenthesis reduces to the constant factor of $\frac{2}{5}$ in $\mathrm{Eq}$. (18) for an isotropic distribution. In anisotropic media this quantity gives the information sought about the moments of the distribution $f(\theta)$. Another experiment is necessary however to separate $\overline{\cos ^{2} \theta}$ from $\overline{\cos ^{4} \theta}$. A measurement of the absorption dichroic ratio $D=\left\langle M_{a z}^{2}\right\rangle /$ $\left\langle M_{a x}^{2}\right\rangle$ would be sufficient as

$$
\overline{\cos ^{2} \theta}=D / D+2, \alpha=0^{\circ} .
$$

Choosing another fluorescence geometry, perhaps with the incident polarization lying in the $X Y$ plane, will also provide a second expression for the moments which can be simultaneously solved along with Eq. (19). Note should be taken that $r$ could range as high as 1.0 in an anisotropic media if perfect order and a zero intramolecular angle $\delta$ obtained.

\section{THE EFFECT OF ROTATIONAL BROWNIAN MOTION}

Molecular disorder and the intramolecular transfer of energy between nonparallel absorption and emission moments have been shown to destroy, at least partially, the polarization of the incident field.

Considerations above were developed under the explicit assumption that the experiment monitors a statistical average over a large collection of molecules statically oriented throughout the lifetime of the fluorescence process. Rotational diffusion of the fluorescent sites will result in a further degradation of the polarization. In this section we will extend the description to include the effects of rotational relaxation.

Due to the anisotropic nature of the absorption process, molecules in certain orientations with respect to the incident beam polarization are more likely to become excited. Polarized illumination therefore has the effect of producing an oriented population of molecules within the medium even when the initial distribution of all molecular axes is random, i.e., inherent in the physics of the experiment, we always deal with a subset of all molecules present in the illuminated volume, those not eliminated through unfavorable orientations in the initial absorption step. In the aligned liquid crystal this photoselection occurs in an already ordered condition of the molecules so that initial anisotropic and isotropic molecular organizations are still distinguishable.

Diffusion complications may be introduced in terms of a time dependent orientational distribution function of the photoselected population $g(\theta ; t)$. The development in time of the orientations of this subset of molecules is governed by the rotational diffusion equation ${ }^{18}$

$$
\partial g / \partial t=D\left[\frac{1}{\sin ^{2} \theta} \frac{\partial^{2}}{\partial \phi^{2}}+\frac{1}{\sin \theta} \frac{\partial}{\partial \theta}\left(\sin \theta \frac{\partial}{\partial \theta}\right)\right] g(\theta, t),
$$

where $D$ is a diffusion constant. A general solution to Eq. (21) is available $e^{8,19}$ and $g(\theta, t)$ can be expressed as

$$
g(\theta, t)=\sum_{l=0}^{\infty} a_{1} e^{-\imath(l+1) D t} P_{l}(\cos \theta)
$$

This expansion is subject to the initial condition that

$$
g(\theta, 0)=M_{a i}^{2} \cdot f(\theta)
$$

for a given degree of order in the gross, unexcited sample, with incident polarized radiation along axis $i$. Equation (23) is simply a statement that from the moment of illumination, the orientational distribution function followed is weighted by the probability of initial absorption $M_{a i}^{2}$. The time evolution of the intensity elements [Eq. (3)] will thus be given by

$$
I_{i j}(t)=\iint M_{e j}^{2} g(\theta, t) P(t) \sin \theta d \theta d \phi,
$$

where $I_{i j}(0)=I_{i j}$, and $P(t)$ is the probability that a molecule emits light at time $t$ during the emission lifetime.

In the formulation of $I_{z z}(t)$, for example, it is clear that

$$
g(\theta, 0)=\cos ^{2} \theta f(\theta),
$$

which implies that $a_{0}=\frac{1}{3} f(\theta), a_{2}=2 a_{0}$, all other $a_{1}$ being null. Hence,

$$
g(\theta, t)=f(\theta)\left[\frac{1}{3}+\frac{2}{3} e^{-t / \tau_{R}} \cdot P_{2}(\cos \theta)\right] \cdot P(t),
$$

where $\tau_{R}=1 / 6 D$ is the rotational relaxation time.

From Eq. (24)

$$
\begin{aligned}
I_{z z}(t)= & \left\{\frac{1}{6} \sin ^{2} \delta \cdot \overline{\sin ^{2} \theta}+\frac{1}{3} \cos ^{2} \delta \cdot \overline{\cos ^{2} \delta}\right. \\
& +e^{-t / \tau} R\left[\sin ^{2} \delta\left(\frac{2}{3} \overline{\cos ^{2} \theta}-\frac{1}{2} \overline{\cos ^{4} \theta}-\frac{1}{6}\right)\right. \\
& \left.\left.+\cos ^{2} \delta\left(\overline{\cos ^{4} \theta}-\frac{1}{3} \overline{\cos ^{2} \theta}\right)\right]\right\} \cdot P(t) .
\end{aligned}
$$

Similarly,

$$
\begin{aligned}
I_{z y}(t) & =\left\{\frac{1}{12} \sin ^{2} \delta\left(2-\overline{\sin ^{2} \theta}\right)+\frac{1}{6} \cos ^{2} \delta \cdot \overline{\sin ^{2} \theta}\right. \\
& +e^{-t / \tau} R\left[\sin ^{2} \delta\left(\frac{1}{4} \overline{\cos ^{4} \theta}+\frac{1}{6} \overline{\cos ^{2} \theta}-\frac{1}{12}\right)\right. \\
& \left.\left.+\cos ^{2} \delta\left(\frac{2}{3} \overline{\cos ^{2} \theta}-\frac{1}{2} \overline{\cos ^{4} \theta}-\frac{1}{6}\right)\right]\right\} \cdot P(t) .
\end{aligned}
$$

Equations $(27,28)$ would describe the decay due to rotational relaxation of the two major fluorescence intensity components in a transient experiment where the sample is illuminated with a pulse of light in a negligibly short period. The usual experiment is, however, one of continuous illumination and the steady state components

$$
\mathrm{I}_{i j}=\int_{0}^{\infty} I_{i j}(t) d t
$$

are required. These may be obtained by averaging Eqs. (27) and (28) over time with a weighting factor $P(t)$ of the form ${ }^{8,20} \tau^{-1} e^{-t / \tau}$, where $\tau$ is the emission lifetime.

Carrying out these integrations, we have shown that

$$
r\left(\tau / \tau_{R} ; \delta\right)=\frac{\bar{P}_{2}(\cos \theta)+\left(\tau / \tau_{R}+1\right)^{-1}\left[\frac{72}{70} \bar{P}_{4}(\cos \theta)+\frac{4}{7} \bar{P}_{2}(\cos \theta)+\frac{2}{5}\right]}{1+2 \cdot\left(\tau / \tau_{R}+1\right)^{-1} \cdot \bar{P}_{2}(\cos \theta)} \cdot P_{2}(\cos \delta)
$$




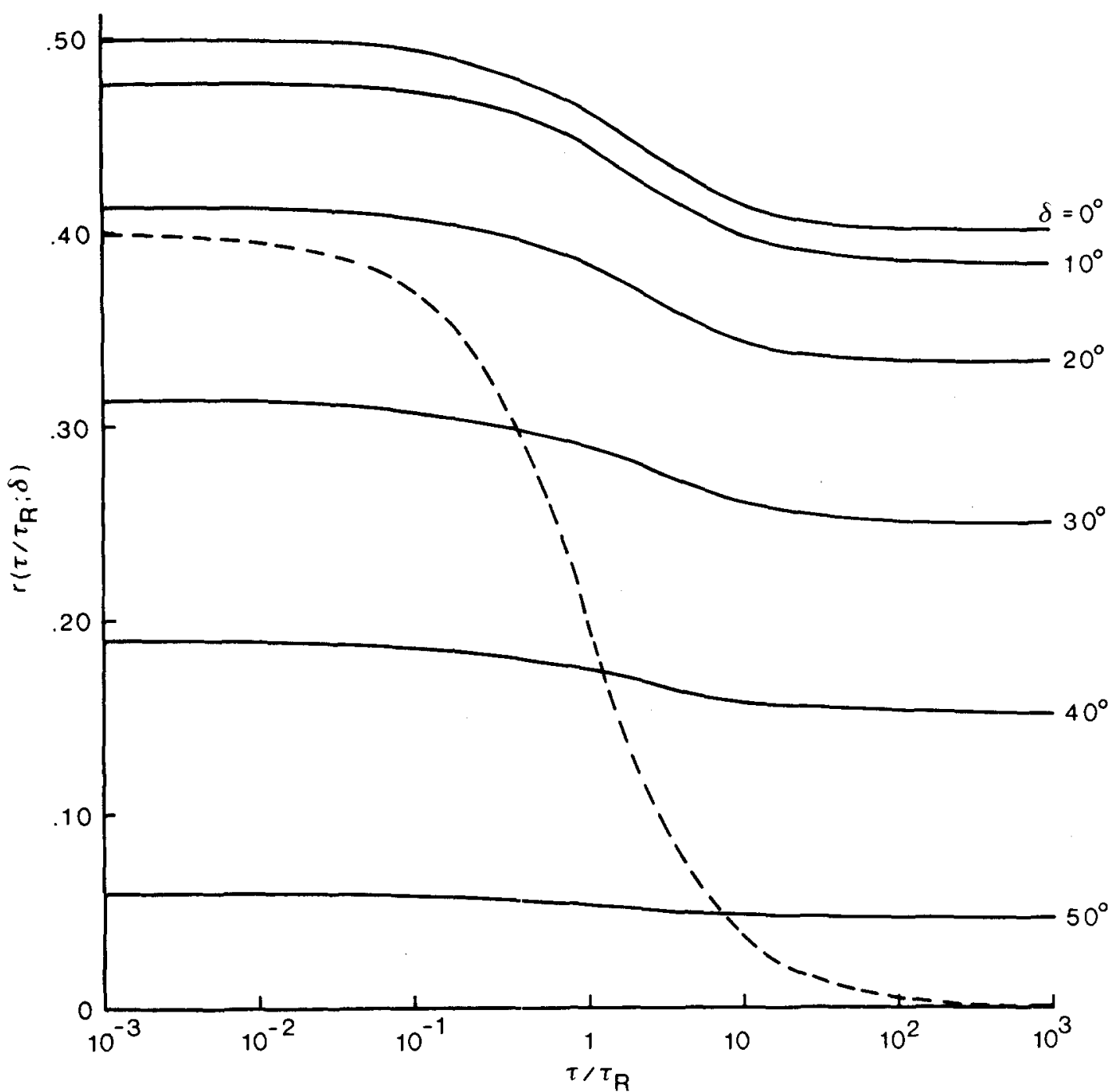

FIG. 3. Anisotropy of fluorescent emission in a uniaxially ordered system as a function of the reduced relaxation time variable $T / \tau_{R}$ for various intramolecular energy transfer angles $\delta$. Solid curves were calculated assuming $\cos ^{2} \theta=0.6, \cos ^{4} \theta=0.4$. The broken curve is Perrin's equation, for the isotropic condition, with $\delta=0^{\circ}$.

If the emission is instantaneous $(\tau=0)$ or the rotational diffusion highly hindered $\left(\tau_{R} \gg \tau\right)$, Eq. (30) reduces to Eq. (19) above for static anisotropic media.

On the other hand, if rotational motion of the probe relaxes out much quicker than the lifetime of the excitation $\left(\tau_{R} \ll \tau\right)$, Eq. (30) becomes

$$
r(\infty ; \delta)=\left\langle P_{2}(\cos \theta)\right\rangle \cdot P_{2}(\cos \delta),
$$

and no information about the fourth moment of the distribution is obtainable. In this case, the fluorescence experiment supplies no more information than an absorption measurement but is complicated by the possible noncoincidence of absorption and emission oscillators of the probe molecules.

In the limit of totally random molecular order, Eq. (30) becomes

$$
r=\frac{2}{5} P_{2}(\cos \delta) \cdot\left(\tau / \tau_{R}+1\right)^{-1}
$$

an expression originally derived by Perrin ${ }^{21}$ to describe depolarization due to Brownian motion of a rotating, spherical molecule in an isotropic fluid.

\section{DISCUSSION}

Figure 3 is a plot of $r\left(\tau / \tau_{R} ; \delta\right)$ versus $\tau / \tau_{R}$ for various values of the molecular angle $\delta$ with reasonable choices of $\overline{\cos ^{2} \theta}=0.6, \overline{\cos ^{4} \theta}=0.4$ for the liquid crystal.

It is clear that a considerable misinterpretation of fluorescent emission data can occur if it is not certain where the experiment falls in the regime of relaxation times and intramolecular energy transfer angles $\delta$. Perrin's Eq. (32), for example, does not apply in liquid crystal systems. Equation (30) is, however, appropriate in such anisotropic media and contains more information about the distribution of molecular order. A faulty approximation to the distribution function will result, however, if the estimates of the $\delta$ and $\tau / \tau_{R}$ operative are substantially in error. A measurement ${ }^{21}$ of the quantity $p_{0}$ to obtain the value of $\delta$ is therefore necessary. If suitable instrumentation is available, transient measurements are also suggested to locate the relaxation region $\left(\tau / \tau_{R} \sim 1\right)$. One possible means of avoiding, or minimizing, the difficulty would be to rapidly quench the sample at each temperature within the mesomorphic range freez- 
ing out thermal motion while preserving the molecular order of the higher temperature phase of interest. This would assure that the experiment is placed on the far left of the curves of Fig. 3.

\section{ACKNOWLEDGMENT}

The authors would like to thank Statens Teknisk-Videnskabelige Forskningsråd, Contract 516-6569. K-339, for partial support of this work.

${ }^{1}$ D. I. Bower, J. Polym. Sci. Polym. Phys. Ed. 10, 2135 (1972).

${ }^{2}$ D. I. Bower, Structure and Properties of Oriented Polymers, edited by I. M. Ward (Applied Science, London, 1975), Chap. 5.

${ }^{3}$ E. B. Priestley and P. S. Pershan, Mol. Cryst. Liq. Cryst. 23, 369 (1973).

${ }^{4}$ S. Jen, N. A. Clark, P. S. Pershan, and E. B. Priestley, Phys. Rev. Lett. 31, 1552 (1973); J. Chem. Phys. 66, 4635 (1977).

${ }_{5}^{5}$ J. P. Heger, J. Phys. (Paris) 36, Lett. 209 (1975).

${ }^{6}$ G. R. Luckhurst and R. Poupko, Chem. Phys. Lett. 29, 191 (1974).

${ }^{7}$ A. C. Albrecht, J. Mol. Spectrosc. 6, 84 (1961).
${ }^{8}$ J. R. Lombardi, J. W. Raymonda, and A. C. Albrecht, J. Chem. Phys. 4, 1148 (1964).

${ }^{9}$ C. R. Desper and I. Kimura, J. Chem. Phys. 38, 4225 (1967).

${ }^{10} \mathrm{~S}$. Nomura, H. Kawai, I. Kimura, and M. Kagiyama, J. Polym. Sci. Part A 8, 383 (1970).

${ }^{11}$ Introduction to Liquid Crystals, edited by E. B. Priestley, P. J. Wojtowicz, and P. Sheng (Plenum, New York, 1975), pp. 72 .

${ }^{12}$ A. Saupe, Z. Naturforsch. Teil A 19, 161 (1964); Angew. Chem. Int. Ed. 61, 947 (1974).

${ }^{13} \mathrm{H}$. Suzuki, Electronic A bsorption Spectra and Geometry of Organic Molecules (Academic, New York, 1967), p. 203.

${ }^{14}$ B. S. Hudson and B. E. Kohler, J. Chem. Phys, 58, 4984 (1973).

${ }^{15}$ Y. Nishijma, J. Polym. Sci, Polym. Symposium 37, 353 (1970).

${ }^{16} \mathrm{G}$. Weber, "Polarization of the Fluorescence of Solutions," Chap. 8 in Fluorescence and Phoshorescence Analysis (Interscience, New York, 1966).

${ }^{17} \mathrm{M}$. Shinitzky, A. C. Dinnoux, C. Gitler, and G. Weber, Biochemistry 10, 2106 (1971).

${ }^{18}$ K. A. Valiev and L. D. Eskin, Opt. Spectrosc. (U.S.S. R.) 12,429 (1962).

${ }^{19} \mathrm{~A}$. Edmonds, Angular Momentum in Quantum Mechanics (Princeton University, Princeton, 1957).

${ }^{20} \mathrm{G}$. Weber, Adv. Protein Chem. 8, 415 (1953).

${ }^{21}$ F. Perrin, J. Phys. (Paris) 7, 390 (1926). 